stained. Because the number of cells staining exceeded the number of heterokaryons and CCL-51 cells in the cultures, they conclude that some unfused MG4 cells were infected and produced progeny mammary tumour virus. On the other hand, when the CCL-51 cells were fused with rat RMG cells, only some 15 to 28 per cent of the heterokaryons and unfused cells stained. This suggests that the rat RMG cells neither induced the increased production of the virus by CCL-51 producer cells nor were productively infected themselves. In other words, the mouse mammary tumour virus seems to be species specific.

In the same issue of the Proceedings (ibid., 742), Scher, Takemoto and Todaro report an interesting attempt to detect supertransformation of 3T3 mouse cells by SV40. It is well known that hamster cells transformed by SV40 can be transformed subsequently by polyoma virus. Scher and his colleagues have therefore asked whether a cell transformed by SV40 can be superinfected and supertransformed by more SV40. They selected lines of SV40 transformed 3T3 cells, which in spite of being transformed grow to comparatively low densities, and infected them with various amounts of SV40 virus to see if the superinfection changed the growth properties of the cells, causing them to grow to greater densities. But such supertransformation did not occur. They then selected lines of SV40 transformed 3T3 cells from which the transforming genome cannot be rescued by fusion with permissive African green monkey cells. These cells were then superinfected with plaque mutant strains of SV40, cultivated for a month or more, and were then fused with monkey cells. If the superinfecting plaque mutant genomes had stably associated with the cell genomes and persisted, they should have been rescuable, but none were. It seems therefore that once a cell has been transformed by SV40, further SV40 virus cannot infect and form a stable association with the genome of the transformed cell.

\section{NUCLEAR PHYSICS}

\section{Polarized Neutron Source}

from a Correspondent

A MUCH needed advance in the technique of producing polarized neutrons has been made simultaneously at the University of Birmingham and Los Alamos. The basis of the development, which will allow the production of neutrons of high polarization over a wide range of energy, is the observation that when polarized deuterons are accelerated on to a deuterium gas target the neutrons produced at zero degrees with respect to the incident beam direction inherit 90 per cent of the vector polarization of the incident deuterons.

It has been known for a long time, of course, that polarized neutrons can be obtained from the $\mathrm{D}(\mathrm{d}, \mathrm{n}) \mathrm{He}^{3}$ reaction even when unpolarized deuterons are used as projectiles. The essence of the present development is that the polarization of the neutrons is independent of energy over a substantial energy range; the Birmingham group reports that the polarization of the neutron is constant over the deuteron energy range of 4 to 16 million electron volts $(\mathrm{MeV})$. A further advantage of the method is that the energy resolution of the outgoing neutron is dependent only on the energy spread of the incident deuteron and the thickness of the gas target. With the recent developments in charged particle polarized beam production it thus seems likely that a beam of $10^{8}$ neutrons per second of 90 per cent polarization will be available in the near future.

What will the experimental physicist do with these neutrons? The quality of data obtained with neutrons as pro- jectiles has always been inferior to the corresponding data obtained with charged particles, and data obtained with polarized neutrons are almost nonexistent. The availability of a source of polarized neutrons thus opens out a completely new field of research. As well as allowing experiments in all branches of nuclear spectroscopy, such a beam of polarized neutrons coupled with a polarized target will enable a detailed study to be made as a function of neutron energy, of the neutronnucleus spin-spin interaction. The ease with which the neutron spin direction can be altered by merely changing the conditions of the deuteron source will be a great boon.

Physicists will no doubt be wondering about where such a facility will become available. At Los Alamos a polarized charged particle ion source is already in operation on the tandem Van de Graaff accelerator. Unfortunately, the University of Birmingham does not have a suitable accelerator to exploit the new technique. It would thus seem a good idea that one of the tandem Van de Graaff laboratories in Britain should adopt the method.

\title{
Mapping the Surface of the Cancer Cell
}

THE chemistry of the surfaces of cells -particularly cancer cells and cells transformed in vitro by tumour viruses - seems destined to become one of the great bandwagons of biology in the next few years. Biochemists and tumour virologists are clearly going to have plenty to chew on and not a few may be expected to retire with indigestion, for it is becoming increasingly clear that transformation to malignancy causes radical but subtle changes in the chemistry and architecture of pre-existing membrane components as well as inducing the synthesis of quite new antigenic determinants. In the past months the changes in the surface architecture which can be monitored by measuring a cell's response to plant agglutinins have excited great interest and the experiments Burger reports next week in Nature New Biology are certain to keep the pot boiling.

He provides convincing evidence that Forssman antigen, a glycolipid with a terminal $\mathrm{N}$-acetylglucosamine residue, exists in a cryptic state in untransformed hamster cells and is exposed as a result of transformation by oncogenic viruses. The fact that transformed cells give a positive reaction in tests for Forssman antigen, whereas comparable untransformed cells do not, is not news. Until now, however, it had always been assumed that transformation somehow switches on the synthesis of this determinant. But that is clearly not the case, for, as Burger shows, in the same way that he showed that agglutinin sites exist but are cryptic in untransformed cells, exposure of untransformed hamster cells to dilute solutions of proteases reveals the same amount of Forssman antigen as does transformation. The antigen must be present in the surfaces of untransformed cells but somehow masked.

Even more intriguing perhaps is that Burger has managed to make a start towards mapping the proximity of this antigen to the receptor sites of various plant agglutinins. He finds, for example, that when Forssman positive cells are exposed to the agglutinin extracted from lentils, Lens culinaris, and then assayed for Forssman antigen the amount of antigenicity detectable is markedly reduced. Pre-exposure to concanavalin $\mathrm{A}$, wheat germ agglutinin or soybean agglutinin, by contrast, has no such blocking effect. The obvious conclusion is that the receptor of lentil agglutinin is close to the Forssman antigen whereas the dispositions of the antigen and the three other agglutinin receptors are unrelated. It does not take much imagination to see where this approach to the architecture of the surfaces of transformed and untransformed cells is leading. It may not be long before there are cell surface maps which, crude though they will undoubtedly be, will nonetheless mark the relative location of a whole series of antigens and other surface markers. 